\title{
Research on the application of color semantics in the human- computer interaction design of smartphones
}

\author{
Bao Xiaoxiao ${ }^{1}$ and Liu Wenming ${ }^{1, \text { a }}$ \\ ${ }^{1}$ School of Design and Art, Shenyang Jianzhu University, 110168 Shenyang, Liao Ning, China
}

\begin{abstract}
To evaluate the user experience in the human-computer interaction design of intelligent products, the quality of color design is a key factor. The purpose of this article is to design the human-computer interaction in smart phones through the study of color semantics, which is one of the branches of product semantics. In particular, the application of color semantics in UI design would help designers better meet user's needs and improve user's experience in the human-computer interaction design of smart products.
\end{abstract}

\section{Introduction}

Nowadays, smart phones have become one of the most important terminals for people to receive information, and the use of smart phones has also become indispensable as a forward position. How can we capture users better and faster and give users more perfect user's experience? Human-computer interaction design has become the key to this problem. In the human-computer interaction and UI design, what does the user first feel ? It must be one of the most important elements in visual information--color. The purpose of this paper is to study one of the branches of product semantics--the application of color semantics in smart phone UI design, so as to help designers to better meet user's needs in human-computer interaction design and improve user's experience. ${ }^{[1]}$

\section{The concept of color semantics}

"Semanteme" originally refers to the meaning contained in the language, "Semantics" is the discipline that studies the meaning of language, and "Product Semantics" refers specifically to the study of the language meaning of a product. It was the designers who combined the knowledge of the study of language and product design.

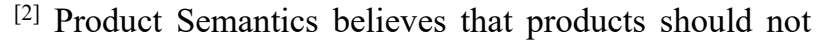
only have physical functions, but also be able to explain or instruct how to use them. At the same time, products should also have a symbolic meaning and can constitute a symbolic environment in people's lives. In other words, product semantics considers not only physical and physiological functions but also psychological, social, and cultural contexts. Its purpose is to make the product a medium for communication and expression, conveying meaning through the use of styling, texture, material, and color.

In the product semantic system, it contains many elements such as form, color, material and technology.
Among these elements, color is the most perceptual element of human visual perception. Color itself is a combination of matter and spirit. It is a perception, a real feeling, not an abstract idea or concept. ${ }^{[3]}$ The color experience begins with the psychological response brought about by the stimulation of light. Therefore, color is a very complex combination of physical and psychological. The process of human perception of color has to go through three phases - physically, psychologically, and culturally, or it is going through three levels -- feeling level, association level, symbol level. Different colors will give people different stimuli, which will lead to different psychological feelings and visual sensations, it may be passionate, depressed, may be warm like in spring or cold like in winter, may be grand and solemn, or light and small; People's life experience will also lead to different emotional associations driven by different colors. Under different cultural backgrounds, colors will also have different cultural symbols.

Color semantics, as the name implies, is to study the significance of color, its core content is: how to accurately convey color information with specific meanings to users in the design, and let users have intuitive and accurate understanding of products through the design colors. Make products not only meet the functional requirements of users, but also meet their psychological needs.

\section{Status of smartphone UI design}

Interaction design is the field of design that defines and designs artificial system behavior. It defines the content and structure of the interaction between two or more interacting individuals so that they can work together to achieve a certain purpose. The goal of interactive system design can be analyzed from two levels __ "usability" and "user's experience", focusing on people-oriented user's needs. UI design is one of the most important

Corresponding author: ${ }^{a}$ Liu Wenming:83373734@qq.com 
aspects of human-computer interaction design. UI design means the design of user interface. It refers to the overall design of the software's human-computer interaction, operation logic, and interface aesthetics ${ }^{[4]}$. It is mainly divided into hardware UI design and software UI design. The scope of the discussion in this paper is smartphone software UI design.

The UI design of smart phones has begun its journey since the invention of the world's first commercial mobile phone in 1973, in the United States, by Martin Coupe, the Motorola engineer. After continuous innovation and development, UI design has gradually become a specialized cross-discipline through comprehensive research on visual experience, psychological experience and erconomics. The style of design has also been gradually transformed from the early "skeuomorphism" design to the "flat" design. ${ }^{[5]}$ It has transformed the human-machine interaction style that simulates natural texture into a simpler color combination style that is more modernistic, which can better improve user's experience and understanding.

\section{Combinations in Design Practice}

In the smartphone UI design, colors are more prominently and intuitively perceived by the user than texts and images. After experiencing different color experiences in daily life, users will accumulate corresponding color semantics, and will also have different physiological and psychological responses to different color stimuli. Therefore, in the UI design of smartphone, it is not only possible to simply stay in the color and aesthetic point of view to design, but to more deeply combine the relationship between color and the product itself and the experience it brings to the user. Then it will achieve the unity of form and function. This paper have divided the application of color semantics in smartphone UI design into three aspects: functional, emotional, and cultural. Through these three different aspects of research and discussion, we hope to help designers more clearly understand the relationship between color semantics and smartphone UI design practices.

\subsection{Functional aspect}

Functional aspect is a top priority in smartphone UI design. When facd with a group of interfaces, how can users find the required information in the first time, learn the direction of the next operation step, and understand what the designer wants to express. All these visual information that users want to understand and designers want to convey is the most important manifestation of functionality in color design. The excellent smartphone UI design can timely convey visual information, accurately express functional semantics, reduce the user's learning cost, and make the interface easier to operate, so that let user feel comfortable and free. The specific requirements mainly include the following points:
First, the interface style must be unified, designers should choose the main color for different products. For example, shopping software usually uses red and orange for their main color, because the color semantics of them are bright, exciting and happy, it can stimulate the user's excitement, so as to achieve the purpose of promotion.

Second, the function partition must be clear, designers should colorize the interface according to the software's different functional groups. In the case of figure 1 , the designer divided different functional areas with color patches of different hue, while the high light area is used for prompting user information, and the low lightness is used for displaying general information.

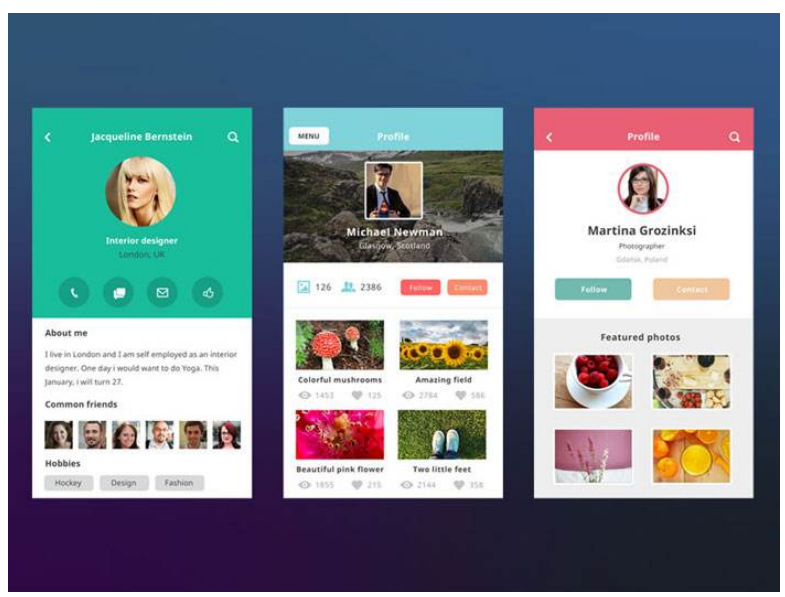

Figure 1. Use different brightness to display information.

Third, the information must be explicitly graded, designers should distinguish colors based on the primary and secondary information content. For instance, in a news and language software with a large amount of information, you can use the repetition, contrast and gradient color changes to mark, distinguish and emphasize information, which enables users to navigate the interface more logically.

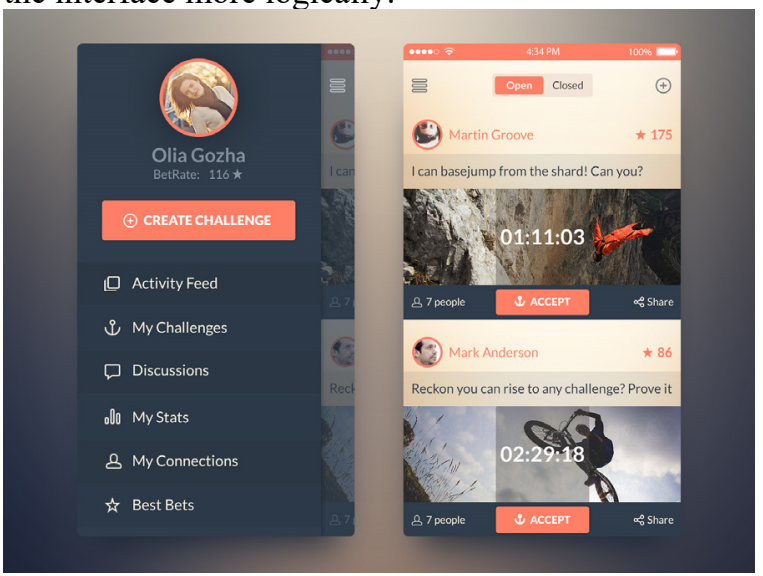

Figure 2. Use color changes to highlight

Fourth, highlight the key modules, according to the color changes the software needs to use. As is shown in figure 2, buttons, guide keys and other modules can be emphasized using contrasting colors, giving the user a visual sensation of jumping to achieve an immediate effect. 


\subsection{Emotional aspect}

"Color evoke emotions, express emotions, and even affect our normal physiological feelings." [6] American art psychologist Blumer said. So, in smartphone UI design, color design needs to focus on the user's emotional factors. Users of different ages and genders have different understandings and feelings about colors. For example, most children prefer bright colors such as red and yellow, black is the most unpopular one. When it comes to adults, most people who prefer blue color are introverted, more literary, and calm down; those who love yellow are outgoing and pursuing ideals; those who love green are mostly decisive and self-respecting; those who prefer red are mostly enthusiastic, impulsive, and enterprising. Therefore, according to the software's target population for the corresponding color design, not only can it better display the software content, but also stimulate the user's psychological resonance, winning the user's heart recognition. In addition, color will also give users psychological feelings such as cold, warm, light, strong and weak, hard and soft, etc.; different hue will lead to a difference between gorgeous and simple feelings; changes in lightness will cause differences in lightness and melancholy; different Purity leads to different feelings of comfort and fatigue. These should all be considered in the UI design of smartphone.

\subsection{Cultural aspect}

According to archeological data, humans in ancient times had already had a certain understanding of color and given different meanings in different colors. With the historical development and accumulation, different colors have gradually become manifestations of different nationalities, regions, and individual lifestyles. That is to say, colors are artificially endowed with nationality, nationality, and regional characteristics, and become an important aspect of human civilization. Components and established a unique color cultural system.

For example, in ancient China, yellow was the exclusive color of the emperor, it represented the center of the azimuth; cyan represented the east of the azimuth and symbolized the righteousness and sacredness; red represented the south and symbolized fiery and happy; black stands for the North, symbolizing righteousness, selflessness; white represents the West, symbolizing killing and silence and so on. But in Western culture, white is a symbol of purity; rose is a representative of passionate love; red represents danger and revolution and war; black even more, represents death and disaster. Therefore, when designers understand the cultural nature of color design, in the UI design of smart phones, it is necessary to select the color of the design according to the user's different cultural attributes.

\section{Summary}

By combining the inherent properties of color with the inter-organizational structure of colors, and the expression of cultural attributes, the color semantics form the ever-changing language form. These linguistic forms exhibit a variety of color properties based on people's different ways of understanding. Color is the most important visual language in the smartphone UI design. In order to achieve the best results while mastering basic color design capabilities, designers must deeply understand the influence of color semantics on user psychology and brand effect, then comprehensively combine all factors for flexible matching. This would greatly make the theme and content of the software more easily to be recognized and memorized by the user, it also brings a higher artistic appeal and the best visual experience to users.

\section{References:}

1. Li Zenhua, Li Xuelin, Li Yuanzhi. J SHENYANG U T, 01, 8-10(2006)

2. Bao Hong, Proceedings of the 2007 International Conference on Industrial Design, Volume 1/2, 3 (2007)

3. Luo Qiao, Design , 15, 36-37(2017)

4. Huang Yafei, J YANGZHOU P C, 15(03), 2123(2011)

5. Sun Minghai, Tan Haoxiang, Design, 07, 3435(2016)

6. Liu Minlu, Li Lun, J YILI NORMAL UNIV (Nat Sci Ed), 04, 77-79(2009) 